\title{
The Pedagogical Model of Philippine STEAM Education: Drawing Implications for the Reengineering of Philippine STEAM Learning Ecosystem
}

\author{
Jovito C. Anito Jr., ${ }^{1, *}$, Marie Paz E. Morales² \\ ${ }^{1}$ Research Office, José Rizal University, Manila, Philippines \\ ${ }^{2}$ Publications Office, Philippine Normal University, Manila, Philippines
}

Received September 15, 2019; Revised October 23, 2019; Accepted November 1, 2019

\begin{abstract}
Copyright $\mathrm{O} 2019$ by authors, all rights reserved. Authors agree that this article remains permanently open access under
\end{abstract} the terms of the Creative Commons Attribution License 4.0 International License

\begin{abstract}
The education sector has to cope with the shifting contour of the global economic landscape, because industries are highly technology-driven, and humans are at the brink of being replaced by machines. New set of skills are required from the human workforce. Higher education institutions (HEIs) need to nurture graduates who demonstrate skills that machines are not capable of. Because most innovations emerge from Science, Technology, Engineering, Agri-Fisheries, and Mathematics (STEAM) disciplines, STEAM education needs to be reinvented. This article proposes a framework to reengineer the Philippine STEAM learning ecosystem, inspired by the recently developed Philippine Pedagogical Model of STEAM Education. The proposed framework was developed as a recommendation to the Philippine government on how the current paradigm and practices of STEAM education in the country can be further improved. The proposed framework features a skills-based learning architecture where competencies are mapped primarily from industry requirements and global trends. The proposed framework specifically implies venturing into non-conventional learning modalities to uphold efficient and effective development of emerging and future skills requirements of the labor market, thereby increasing its productivity and innovation indices.
\end{abstract}

Keywords STEAM Education, Future Education, Education 4.0, Philippines

\section{Introduction}

This article primarily aims to extend the recently developed Pedagogical Model of Philippine STEAM
Education (Morales, Anito, Avilla, Sarmiento, et al., 2019), which hinted an archaic paradigm on Science, Technology, Engineering, Agri-Fisheries, and Mathematics (STEAM) education. Because STEAM is a significant driver of a country's national economy and global competitiveness, the STEAM learning ecosystem has to be reinvented.

The 2018-2019 Global Competitiveness Report revealed that the Philippine competitiveness index increased by 2.3 points, scoring positively in all individual competitiveness pillars (Schwab, 2018). While this clearly shows improvement of Philippine competitiveness metrics, the 2018 Global Innovation Index (GII) ranked the country at $62^{\text {nd }}$ out of 126 economies in terms of Innovation Efficiency Ratio, down from $55^{\text {th }}$ and $49^{\text {th }}$ from the 2017 and 2016 respectively (World Intellectual Property Organization, 2018). As we owe much of the innovations and inventions to STEAM practitioners and educators, lagging behind in terms of innovation indicates a weak scientific workforce. Based on UNESCO recommendation of 380 scientists per million, Philippines registers at 189, lagging behind its ASEAN neighbors - Vietnam at 674, Thailand at 974, and Malaysia at 2,100 per million (Jalea, 2018). The scarcity of scientists in the country is attributed to the shortage of STEAM graduates. The Philippine Commission on Higher Education (CHED) data revealed that STEAM programs register a meager completion rate of 21\% (Morales, Anito, Avilla, Sarmiento, et al., 2019). Apparently, this calls for an intensive effort to rethink about the country's STEAM education vis-à-vis the labor market conditions and Philippines' global position in terms of competitiveness and innovation. Responding to this challenge, CHED through the Philippine Normal University conducted a study which investigated the perspectives and practices of Philippine HEIs on STEAM education (Morales, Anito, Avilla, Sarmiento, et al., 2019). 
The research project developed a self-rating tool for STEAM proficiency indicator of educators (Morales, Anito, Avilla, Abulon, \& Palisoc, 2019) and the Philippine STEAM Education Model (Morales, Anito, Avilla, Sarmiento, et al., 2019), a component of which is the Pedagogical Model of Philippine STEAM Education (PMPSE).

\section{Methods}

The Pedagogical Model of Philippine STEAM Education was developed through a three-tier model-building qualitative data analysis. The study involved 33 HEIs sampled from all over the country. Data were culled primarily from interview of HEI top-level administrators (Presidents and Vice Presidents), mid-level administrators (deans, coordinators, department heads), and teachers. Actual observation of classes was also conducted, and sample lesson plans of STEAM teachers observed were analyzed. A total of 99 interview transcripts and 99 sets of observation notes and sample lesson plans were analyzed in the development of the model.

The analysis advanced following a divergent grounded theory method (Amsteus, 2014). Specifically, while the research method aligned more with the constructivist grounded theory (Charmaz, 2017), its primary principle on iterative data collection and analysis was not followed. The administrative and logistics constraints of the project compelled the researchers (including the authors of this article) to diversify the analysis method while maintaining the overall quality and validity of the process. Specifically, the researchers employed a multi-level reflexivity mechanism data collection, data analysis, and quality audit. The analysis of data followed the usual conceptualization method of constructivist grounded theory comprising coding (descriptive and analytical) and memoing through a qualitative data analysis (QDA) software.

The first level coding utilized the interview transcripts. Following the coding and memoing procedures, a code system was established containing the codes, categories, and memos as complete documentation of code naming, code evolution, and code dynamics along with the properties and dimensions of codes and the code system itself. Memos of the first-tier analysis were subjected into another round of coding and memoing (Tier 2 coding), both as a form of quality audit and as a means to enrich the emerging model. The last tier utilized the code system developed in the previous tier as a framework to capture the indicators from observation notes and sample lesson plans.

\section{Results}

\subsection{The Pedagogical Model of Philippine STEAM Education}

The Pedagogical Model of Philippine STEAM
Education (PMPSE) articulates the interdependence between the institutional pedagogical culture and its STEAM pedagogical processes. The pedagogical culture refers to the institution's mechanism of planning, disseminating, and evaluating its pedagogical processes and the extent by which the research and teaching nexus is advanced (Morales, Anito, Avilla, Sarmiento, et al., 2019). PMPSE underscored that HEIs shape the teaching and learning practices, which in return significantly measures institutional pedagogical culture. PMPSE argued that STEAM education should produce graduates who possess the discipline-based competencies (Morales, Anito, Avilla, Sarmiento, et al., 2019).

The Philippine higher education defines discipline-based competencies primarily as the competencies prescribed by the Commission on Higher Education through its Policies, Standards, and Programs (PSGs). Review of CHED PSGs for STEAM disciplines (Morales, Anito, Avilla, Abulon, et al., 2019) suggests that these competencies may no longer be what the current and future industries demand. The next section elaborates the PMPSE vis-à-vis the emerging competencies.

\subsection{The Pedagogical Model for Philippine STEAM Education}

As emphasized in the previous section of this article, the PMPSE illustrates the current pedagogical perspectives, ideals, and practices of Philippine HEIs on STEAM. However, the possibilities, opportunities, and disruptions brought about by the Fourth Industrial Revolution (4IR) on business and industry demands a relatively radical shift in educational contour. The educational setting in the 4IR must address the challenges posed by the rise of smart machines, artificial intelligence, big data analytics, and virtual/augmented reality (Benešova \& Tupa, 2017) among others. While this technological advancement creates opportunities for business and industries, it also threatens a new divergence and polarization within and between economies and societies (Schwab, 2018). The rapid shift in industrial processes from work performed by humans to those performed by machines and algorithms results to major transformations in the labor market (World Economic Forum, 2018). In response to this, the education sector is expected to help reconfigure the workforce by ensuring that competencies developed among its learners are responsive to the demands of the changing landscape of the world of work. Because education embeds skills and competencies in the labor force (Schwab, 2018), it is deemed crucial in balancing the impact of the technological advances on economic and social infrastructure. This suggests that higher education institutions need to reconceive their programs, especially on STEAM education, to ensure that graduates possess the skills necessary in the 4IR.

The PMPSE was further examined vis-a-vis the skills 
requirements of the 4IR. The researchers looked into how the current pedagogical model be improved to ensure alignment of Philippine STEAM programs with the skills requirements of businesses and industries in the 4IR. The complexity of the machine-dominated era demands that graduates possess abilities that are unique to human beings in order to be relevant. Education therefore must see to it that graduates have comparative advantage over machines such that humans are able to control them, which apparently, only the qualified and highly-educated workers are able to do so (Benešova \& Tupa, 2017). Schwab (2018) argued that highly-educated populations are more productive because they possess greater collective ability to perform tasks, can transfer knowledge quickly, and create new knowledge and applications. Studies establish that the 4IR requires workers who possess social and personal competencies such as sense-making, social and emotional intelligence, and cross-cultural competency (Bughin, et al., 2018; Davies, Fidler, \& Gorbis, 2011; World Economic Forum, 2018; Xing \& Marwala, 2017). The same studies furthered that workers also need to possess technical and methodological skills such as new media literacy, cognitive flexibility and transdisciplinarity, design mindset, virtual collaboration, and critical, novel, adaptive, and computational thinking. This suggests that the pedagogical and curricular efforts of a higher education institution must ensure development and enhancement of these skills among its graduates.

\subsubsection{Institutional Pedagogical Culture}

The PMPSE illustrated how the pedagogical culture of an institution affects its STEAM programs. Specifically, it postulates the need to establish an institutional mechanism for periodic curriculum review, alignment of faculty specialization with content taught, school, industry, and community partnership, and dissemination of pedagogy. Aligning with the conditions and requirements of the 4IR, curriculum review for Philippine STEAM education must focus on identifying the key work competencies of graduates that satisfy the requirements of the labor market. Philippine HEIs must also look for expertise beyond its physical space. 4IR is an era wherein specializations converge online. This means that Philippine HEIs can outsource the teaching of specialized content from other forms and agents of learning, especially those in the virtual space. As Shahroom and Hussin (2018) argued, HEIs as residential institutions will not survive in the 4IR. The physical space like buildings may be hopelessly unsuited and no longer needed (Shahroom \& Hussin, 2018). Education can take any form and so must schools. The question of whether a faculty member with a graduate degree in Biology can effectively teach Physics is no longer pertinent. What is more relevant to ask is whether the institution has a mechanism to take advantage of the wide array of content and expertise available in the virtual learning communities and other learning media outside the school's physical space. The 4IR necessitates HEIs to embrace learning across formal, non-formal, and informal avenues (Chao, 2017). The PMPSE specifically articulates that teachers with industry experience are preferred to teach STEAM courses. This suggests that STEAM teachers in the 4IR need not be exclusively STEAM teachers in an HEI. They can be primarily industry workers who opt to offer authentic learning by sharing their experiences on the application of STEAM, either in an e-learning platform or in a face-to-face setting.

In establishing and sustaining partnership with the industry and community, the Philippine STEAM pedagogy must address the major transformation of the business, government, industrial, and social infrastructures brought about by smart machines and process automation. School-industry-community partnership entails seamless access of relevant data across these sectors such that each sector acts according to the conditions and requirements of the others. For example, HEIs can evaluate the quality and relevance of its programs through the data collected from the industry and community partners. HEIs must utilize these data in reconfiguring the curriculum. Through data, HEIs can uncover relationships and patterns to model quality and relevant learning architecture.

Support to pedagogy is also highlighted in the PMPSE. The 4IR is characterized by taut competition of and for talents such that low-skilled workers will be displaced (Bughin, et al., 2018). This implies that as HEIs develop among learners the skills relevant to the 4IR, STEAM teachers must first possess the relevant knowledge and skills. This calls for an accelerated reskilling and upskilling of STEAM teachers to be retained in the academe. Further, HEIs must see to it that STEAM teachers are trained in an interdisciplinary environment where scientists understand arts and humanities and vice versa (Xing \& Marwala, 2017).

\subsubsection{The Teacher Pedagogical Character}

Philippine STEAM teachers in the 4IR are envisioned to exude a non-restrictive epistemological stance. They demonstrate transdisciplinarity and cognitive flexibility. Transdisciplinarity refers to one's literacy in and ability to understand concepts across multiple disciplines (Davies, Fidler, \& Gorbis, 2011). Cognitive flexibility (Xing \& Marwala, 2017) pertains to one's ability to converse in the language of a broader range of disciplines. The transdisciplinary character of STEAM teachers must likewise manifest in their research activities. STEAM teachers must engage in transdisciplinary research projects. Building upon STEAM and STEAM Education researches as proposed by the PMPSE, the research projects of STEAM teachers in the 4IR extends beyond the disciplinary boundaries of STEAM and STEAM education. 


\subsubsection{The STEAM Pedagogical Processes}

The PMPSE advances context-based learning where STEAM concepts are presented through simulations and real-life applications. Teaching STEAM in the 4IR requires an authentic learning experience where application of STEAM concepts is borderless. Authentic learning includes learning using technology to provide learners the real-world learning experiences (Knowledge Works, 2012). Learning technology includes virtual simulations, games, collaboration and social media tools. Authentic learning also entails digital apprenticeship (Knowledge Works, 2012). STEAM learning in the 4IR is characterized by learning for and with the machines. Learning with the machines underscore the codependence of humans and machines. STEAM education then must provide learners a wide range of experiences where machines augment human capabilities in a dynamic venture. Learning for the machines emphasize the need for humans to understand the nature of machines to be able to control them. Assessment of STEAM pedagogy in the 4IR is well defined. It encompasses all categories of learner data from which pattern of behavior and understanding are drawn. Learner data may even include gestures, vocal tone, and other information drawn from sensors which can be codified and modeled.

\subsubsection{The Outcomes of STEAM Education}

The PMPSE presents the characteristics of STEAM graduates as competent professionals, productive citizens, and critical thinkers. Competent STEAM professionals demonstrate social, emotional, and cross-cultural intelligence. Social intelligence is fundamental to collaborations. It is the ability to connect to others in a deep and direct way, to sense and stimulate reactions and desired interactions (Davies, Fidler, \& Gorbis, 2011) and to persuade and negotiate (World Economic Forum, 2018). Emotional intelligence pertains to empathy and adaptability (Bughin, et al., 2018) which are crucial in people management (Xing \& Marwala, 2017). Cross-cultural intelligence is the ability to operate in different cultural settings and identify and communicate points of connection such as shared goals, priorities, and values (Davies, Fidler, \& Gorbis, 2011).

Productive STEAM professionals exhibit novel and adaptive thinking, computational thinking, literacy in new media, design mindset, and virtual collaboration. Novel and adaptive thinking is the proficiency at thinking and coming up with solutions and responses beyond that which is rote or rules-based (Davies, Fidler, \& Gorbis, 2011). Adaptive thinking requires creativity, originality, initiative (World Economic Forum, 2018) complex information processing and interpretation, decision making (Bughin, et al., 2018), and critical thinking (Xing \& Marwala, 2017). Computational thinking is the ability to translate vast amounts of data into abstract concepts and to understand data-based reasoning (Davies, Fidler, \& Gorbis, 2011). New media literacy is the ability to critically assess and develop content that uses new media forms and to leverage these media for persuasive communication (Davies, Fidler, \& Gorbis, 2011). Design mindset is the ability to represent and develop tasks and work processes for desired outcomes (Davies, Fidler, \& Gorbis, 2011). Design mindset also involves creativity, originality, initiative, critical thinking (World Economic Forum, 2018) as well as advanced IT skills, programming, engineering, and technology design (Bughin, et al., 2018). Productive STEAM professionals are also able to work productively, drive engagement, and demonstrate presence as a member of a virtual team (Davies, Fidler, \& Gorbis, 2011).

Critical STEAM professionals demonstrate adeptness in sense making, cognitive flexibility, and cognitive load management. Sense-making is the ability to determine the deeper meaning or significance of what is being expressed (Davies, Fidler, \& Gorbis, 2011). Cognitive flexibility is the ability to think about multiple concepts, theories, and disciplines simultaneously (Xing \& Marwala, 2017). It is closely linked to transdisciplinarity, the literacy in and ability to understand concepts across multiple disciplines (Davies, Fidler, \& Gorbis, 2011).

The changing landscape in education brought about by the Fourth Industrial Revolution necessitates HEIs to introduce radical shifts in the curriculum, especially in STEAM education. HEIs need to reconceive their business ecosystems, re-identify their competitive edges, and rebuild their service architecture (Xing \& Marwala, 2017). While PMPSE hints an antiquated learning paradigm, Philippine HEIs may begin its transformation of STEAM education by

- introducing inter- and multi-disciplinary degrees;

- advancing an appropriate blend of learning models (e.g. blended learning, MOOCs)

- embracing the emergence of Internet of Everything; demands

- $\quad$ integrating routine education activities into software;

- updating the digital infrastructure; and

- enhancing connectivity among all parties residing in higher education value chain (Xing \& Marwala, 2017).

Philippine HEIs with reputable STEAM programs may also consider venturing into franchise programs (Xing \& Marwala, 2017) where they accredit and authorize another HEI to deliver their STEAM courses/programs. This may entail collaborative training, reskilling, and upskilling. This is to ensure that quality and relevance of STEAM education, as demonstrated by these reputable HEIs, are diffused across other institutions.

Government support is deemed crucial in the transition of Philippine HEIs from the traditional STEAM learning modality to a future-proof and 4IR-aligned STEAM learning model. For example, CHED may revisit its 
Policies, Standards, and Guidelines (PSGs) to elucidate policies, standards, and guidelines for interdisciplinary and multidisciplinary degrees. CHED may also embrace diverse forms of credentials, certificates, and reputation markers (Knowledge Works, 2012) and provide guidelines for micro certification and similar schemes.

\subsection{Framework for the Reengineering of the Philippine STEAM Education}

Informed by the archaic view of STEAM learning as hinted by the PMPSE, this article presents the framework (Fig. 1) for the reengineering of Philippine STEAM education towards raising our country's position in competitiveness and innovation metrics.

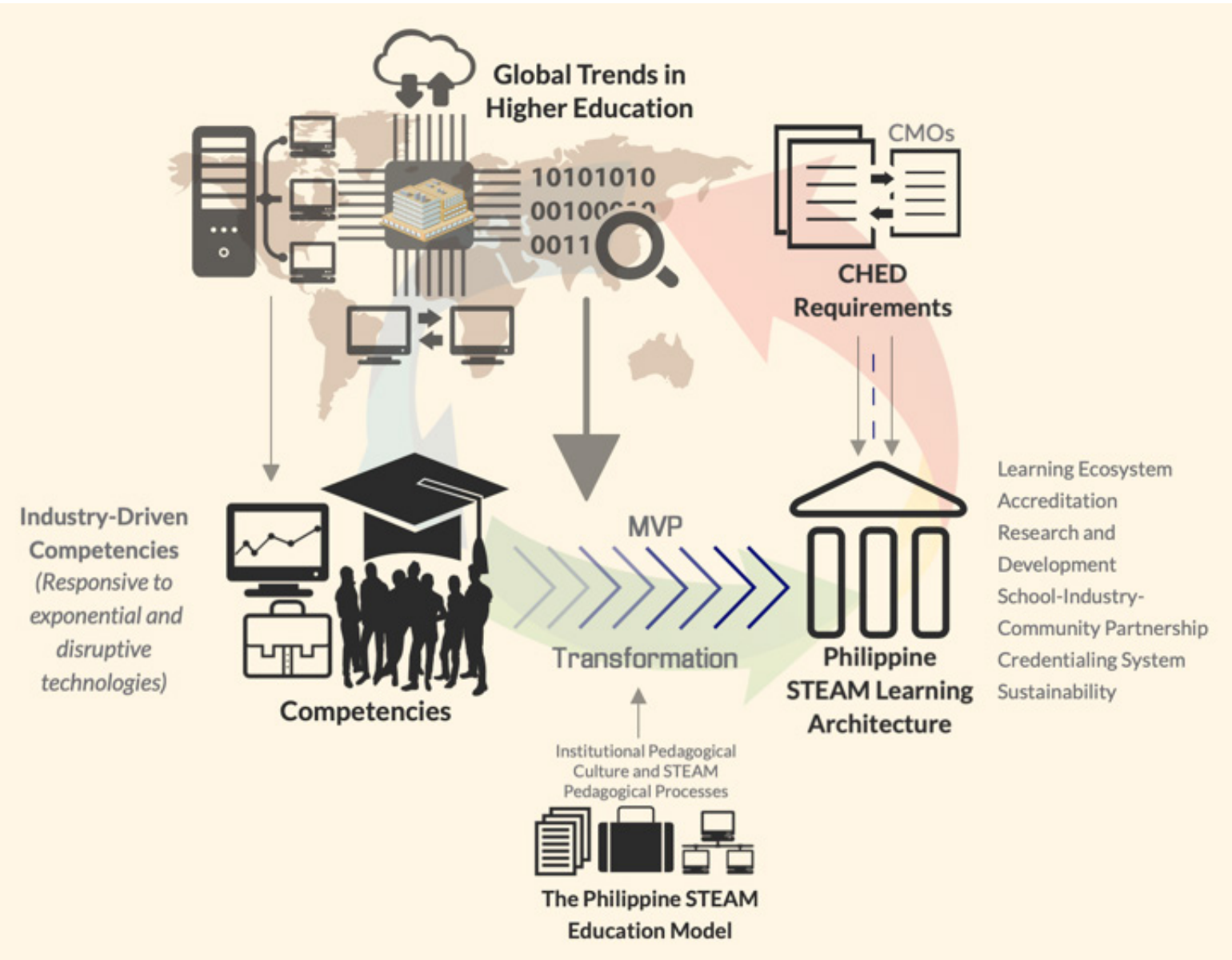

Figure 1. Framework for the reengineering of Philippine STEAM Education 
The proposed framework highlights an analysis of skills rooted on actual needs of industries and not on state-prescribed standards. Driven by the changes in global economic and economic landscape brought about by disruptive technologies, the framework proposes to reengineer the Philippine STEAM learning architecture to balance the disruption. Learning ecosystem pertains to program typology and infrastructure such as modality, and learning space. It specifically answers questions pertaining to STEAM teaching and learning, including learning assessment. Accreditation refers to the position of the new STEAM learning ecosystem relative to local and international accreditation and equivalency standards. Research and development refers to the nexus between research, learning, innovation, and development as an inherent feature of the Philippine STEAM education. Credentialing system refers to the process of establishing reputation and proficiency across programs. Sustainability refers to the mechanism to uphold ethical implementation of the new academic programs especially in managing big data. The proposed framework specifically aims to:

1. model expert forecasts of Philippine STEAM education in terms of:

a. learning ecosystem (curricular identity; modality; learning space; talent and skill acquisition; assessment; professoriate; stacking, unstacking, and hypersegmentation; and accelerated continuous professional development)

b. accreditation (international standards, local constraints)

c. research and development (research, learning, innovation, development, extension)

d. school-industry-community partnership

e. credentialing system (reputation markers, certificates)

f. sustainability (funding, ethics and information security, forecasts, green initiatives)

2. map the competency requirements of industries;

3. model global forecasts on higher education;

4. map the competency alignment/mismatch between industry requirements and current model of Philippine STEAM Education;

5. design the Philippine STEAM Learning Architecture in terms of:

a. learning ecosystem (curricular identity; modality; learning space; talent and skill acquisition; assessment; professoriate; stacking, unstacking, and hypersegmentation; and accelerated continuous professional development)

b. accreditation (international standards, local constraints)

c. research and development (research, learning, innovation, development, extension)

d. school-industry-community partnership

e. credentialing system (reputation markers, certificates) f. sustainability (funding, ethics and information security, forecasts, green initiatives)

6. develop Prototype Programs, which model completely new programs for STEAM, anchored on the new Philippine STEAM Learning Architecture;

7. test and model Viable Programs anchored on the new Philippine STEAM Learning Architecture;

8. develop user manuals and protocols for the development and testing of Viable STEAM Programs anchored on the Philippine STEAM Learning Architecture;

9. conduct institutional information dissemination and capacity-building programs on the development and testing of Viable Programs

\subsubsection{The Proposed Methods in Reengineering the Philippine STEAM Education}

The proposed framework will be carried out as a formal research primarily employing Delphi Survey Method to map the current and emerging competency requirements of industries as inputs in the design a future-proof STEAM learning architecture. A panel of experts for the Delphi Survey will be invited from industry organizations, human resource organizations, and talent and recruitment groups. An analysis of job announcements among top business and industries in the country and abroad will also be conducted to map the skills required by their business processes. Literature synthesis and document analysis will likewise be conducted to explore the global trends and forecasts of higher education. A survey on students' experience with the current STEAM pedagogical practices in Philippine HEIs will also be conducted. Another survey will be conducted to examine the institutional provisions and study conditions in the area of STEAM education. The study will end with a series of national dialogues and for a with various stakeholders.

A. Competencies Mapping - generally aims to conduct a comprehensive documentation of future-driven skills. The following strategies will be employed to attain this:

(a) Delphi Survey - Expert participants are from the Department of Science and Technology (i.e. Sectoral Planning Councils, R\&D Institutes, National Academy of Science and Technology, National Research Council of the Philippines), Industry Organizations, and Human Resource Associations (e.g. IT Business Process Association of the Philippines, People Management Association of the Philippines, Accenture, Jobstreet). The Delphi survey will focus on expert forecasts on STEAM Education alignment with industry-driven and future-driven competencies.

(b) Document Review - Job Announcements/Hiring Calls from Jobstreet, Accenture, LinkedIn and other major recruitment and talent and human resource management companies will be analyzed to map the competencies required by specific companies. Documents pertaining to global trends and forecasts in STEAM education across all platforms and modalities such as the alternative 
universities will likewise be examined to draw out specific skills being focused in their programs.

(c) Systematic Literature Review - Literature Review from future-oriented Journals will likewise be reviewed to extract the trends and forecasts in STEAM education. Inclusion criteria for the systematic review include STEAM Education, Education of the Future, Future of Higher Education, among others. Literature and data on Philippine graduate employability will likewise be explored to examine the gaps in skills required in specific jobs and skills developed in Philippine HEIs.

(d) Student Experience Survey - A survey among HEI graduates will be conducted to examine the alignment of skills developed/learned from HEIs or TESDA trainings and the skills required by specific jobs.

B. Design of the STEAM Learning Architecture generally aims to consolidate the data drawn from the Delphi Survey, Document Review, Systematic Literature Review, and Student Experience Survey and translate them into a Learning Architecture focused on the following components:

1. learning ecosystem (curricular identity; modality; learning space; talent and skill acquisition; assessment; professoriate; stacking, unstacking, and hypersegmentation; and accelerated continuous professional development);

2. accreditation (international standards, local constraints);

3. research and development (research, learning, innovation, development, extension);

4. school-industry-community partnership;

5. credentialing system (reputation markers, certificates);

6. sustainability (funding, ethics and information security, forecasts, green initiatives).

C. Design of Viable STEAM Programs - generally aims to translate the learning architecture into specific programs, tested and validated through Minimum Viable Products (MVP). The MVP process will be specifically conducted through a series of meetings and presentations with industry partners. The prototype programs will be packaged in a form of a resume, showcasing the competencies of STEAM graduates following the new STEAM program architecture. The MVP meetings and presentations will revolve around whether industry representatives will hire graduates with such resume of competencies. Inputs from these meetings and presentations will serve as inputs for the enhancement of the prototype programs until minimum viable programs are achieved.

Reengineering the Philippine STEAM education will specifically benefit the following groups and sectors:

1. The Philippine Higher Education Sector - This project endeavors to map the industry-driven and future-driven competencies to provide policy inputs to CHED on STEAM Learning Architecture that specifically aligns with future industry requirements. The forecasting nature of Delphi survey allows predicted STEAM learning contexts to emerge and to be integrated in the design of viable STEAM programs.

2. Philippine HEIs - The manual on developing Viable STEAM Programs is specifically helpful in reengineering the STEAM programs among HEIs such that STEAM programs will specifically satisfy the demands of the future industry sector. The efficiency of Viable STEAM programs are likewise envisioned as a cost-cutting strategy of HEIs where quality of STEAM learning is boosted at a reduced operations cost.

3. STEAM teachers - The new learning architecture is envisioned to strengthen the blend between online and face-to-face decongesting modalities of learning. Process automation is likewise predicted in many areas such as assessment and certification, thereby reducing the workload of teachers.

4. STEAM Learners - The reengineered STEAM learning ecosystem is envisioned to advance customized learning methods such that learning potential of each learner is maximized.

5. The Science and Technology Sector (DOST) and the Philippines - Reengineering the Philippine STEAM Learning Architecture aim to provide quality STEAM education in the country thereby improving national productivity and economy. Quality STEAM education likewise aims to attract and retain smart and STEAM-driven students thereby increasing the number of scientists and STEAM competent professionals in the country. Scientists and STEAM professionals are considered as the key actors in all technological advancements and innovations across different sectors consequently accelerating economic growth.

\section{Conclusions}

This article describes the results of the state-funded project which tendered a pedagogical model of Philippine STEAM education. As elucidated in the model, the Philippine STEAM HEIs view STEAM learning more as an institution- and teacher-dependent endeavor rather than a learner-empowerment and life-long learning process. As can be drawn from the analysis, Philippine HEIs may advance by focusing on the development of key work competencies that satisfy the current and anticipate the emerging and future requirements of the labor market. Philippine HEIs may likewise explore a more fluid and multidisciplinary infrastructure of talents and expertise and embrace non-conventional learning modalities. STEAM educators in Philippine HEIs may likewise engage in transdisciplinary research projects, ensure borderless application of STEAM, expose learners to 
transdisciplinary problems, and ensure development of relevant skills among learners. Responding to this, a framework towards reengineering of Philippine STEAM learning ecosystem has been proposed wherein mapping the competencies is primarily anchored on actual industry requirements and not on state-prescribed competency standards. The framework primarily aims to reinvent Philippine STEAM education in terms of learning ecosystem, accreditation, research and development, school-industry-community partnership, credentialing system, and sustainability. The Viable Programs anchored on this new STEAM learning architecture are envisioned as drivers of the Philippines' global position in terms of higher education trends, competitiveness, innovation, and economic metrics.

\section{Acknowledgements}

The authors would like to thank the Philippine Commission on Higher Education (CHED), the funding agency of the research project described in this article.

\section{REFERENCES}

[1] Amsteus, M. N. (2014). The validity of divergent grounded theory method. International Journal of Qualitative Methods, 71-87.

[2] Benešová, A., \& Tupa, J. (2017). Requirements for education and qualification of people in industry 4.0. International Conference on Flexible Automation and Intelligent Manufacturing (pp. 2195-2202). Italy: Prcedia Manufacturing.

[3] Bughin, J., Hazan, E., Lund, S., Dahlstrom, P., Wiesinger, A., \& Subramaniam, A. (2018). Skill shift automation and the future of the workforce. Brussels: McKinsey Global Institute.

[4] Chao, R. J. (2017, November 10). Educating for the fourth industrial revolution. University World News.

[5] Charmaz, K. (2017). Special invited paper: Continuities, contradictions, and critical inquiry in Grounded Theory. International Journal of Qualitative Methods, 1-8.

[6] Davies, A., Fidler, D., \& Gorbis, M. (2011). Future Work Skills 2020. CA: Institute for the Future for the University of Phoenix Research Institute.

[7] Jalea, M. (2018, March 31). PH needs 19,000 more scientists, researchers. The Manila Times.

[8] Knowledge Works. (2012, September 1). A glimpse into the future of learning. Retrieved from Knowledge Works: https://knowledgeworks.org/resources/forecast-3/

[9] Morales, M., Anito, J., Avilla, R., Sarmiento, C., Palisoc, C., Elipane, L., Palomar, B. (2019). The Philippine STEAM Education Model. Manila: Philippine Normal University.
[10] Morales, M., Anito, J., Avilla, R., Abulon, E., \& Palisoc, C. (2019). Proficiency indicators for Philippine STEAM (Science, Technology, Engineering, Agri/fisheries, Mathematics) educators. Philippine Journal of Science, 265-281.

[11] Schwab, K. (2018). The Global Competitiveness Report 2018. Geneva, Switzerland: World Economic Forum.

[12] Shahroom, A., \& Hussin, N. (2018). Industrial revolution 4.0 and education. International Journal of Academic Research in Business and Social Sciences, 314-319.

[13] World Economic Forum. (2018). The future of jobs report 2018. Geneva, Switzerland: World Economic Forum Centre for the New Economy and Society.

[14] World Intellectual Property Organization. (2018). Global Innovation Index 2018: The Philippines. Retrieved from World Intellectual Property Organization:https://www.wip o.int/edocs/pubdocs/en/wipo_pub_gii_2018-profile36.pdf

[15] Xing, B., \& Marwala, T. (2017). Implications of the fourth industrial age on higher education. Retrieved from The Thinker: http://arxiv.org/abs/1703.09643 